\title{
Incorporação do dióxido de titânio como aditivo em membranas de poliamidas
}

\author{
Titanium dioxide incorporation as an additive in polyamides membranes \\ Incorporación de dióxido de titanio como aditivo en membranas de poliamidas
}

Recebido: 21/06/2021 | Revisado: 30/06/2021 | Aceito: 06/07/2021 | Publicado: 16/07/2021

Larissa Dias Rebouças

ORCID: https://orcid.org/0000-0003-3890-6572 Universidade Estadual da Paraíba, Brasil E-mail: larirbcs@gmail.com

Bruna Aline Araújo

ORCID: https://orcid.org/0000-0002-9429-6994 Universidade Estadual da Paraíba, Brasil

E-mail: brunaaaraujo15@gmail.com

José Everton Soares de Souza

ORCID: https://orcid.org/0000-0003-0731-534X Universidade Estadual da Paraíba, Brasil

E-mail: everton_g3@hotmail.com

Kênia Kelly Freitas Sarmento

ORCID: https://orcid.org/0000-0001-6815-9577 Universidade Estadual da Paraíba, Brasil

E-mail: keniakellys41@gmail.com

Carlos Antônio Pereira de Lima

ORCID: https://orcid.org/0000-0002-1301-6066 Universidade Estadual da Paraíba, Brasil E-mail: caplima@uepb.edu.br

Keila Machado de Medeiros

ORCID: https://orcid.org/0000-0001-9250-1432

Universidade Federal do Recôncavo da Bahia, Brasil

E-mail: keilamedeiros@ufrb.edu.br

\begin{abstract}
Resumo
Dentre as tecnologias de tratamento avançados de efluentes, os processos de separação por membranas se destacam pela abrangência de aplicações, dentre elas dessalinização de águas, indústria e tratamento de efluentes. As nanopartículas utilizadas como aditivos, ao serem incorporadas durante a preparação da solução polimérica para a obtenção das membranas, podem trazer melhorias ao desempenho e eficácia das membranas como meios filtrantes, formando assim membranas híbridas. Este estudo visa revisar os principais estudos envolvendo o dióxido de titânio $\left(\mathrm{TiO}_{2}\right)$ como aditivo em membranas poliméricas, e apresentar seus efeitos e seu comportamento ao ser incorporado, de acordo com os métodos e técnicas utilizadas na literatura recente. Em especial, as membranas de poliamidas têm sido amplamente estudadas e utilizadas tanto em sua forma pura quanto com a adição de um ou mais aditivos, que podem conferir melhorias importantes, como redução de rugosidade aumenta de porosidade, correção de defeitos, melhoria da permeabilidade, dentre outros. Portanto, o $\mathrm{TiO}_{2}$ é um dos aditivos que se destacam como opção para incrementar a porosidade, permeabilidade, hidrofilicidade e reduzir o efeito de incrustação destas membranas. Assim, sendo, pelas pesquisas experimentais realizadas ficou constatado que esta carga é largamente utilizada como aditivo na obtenção de membranas a partir de poliamidas por possuir características desejáveis nos processos de separação, se apresentando como opção de um material híbrido avançado para aplicação no tratamento de diversos efluentes.

Palavras-chave: Membranas poliméricas; Composto inorgânico; Híbridos; Tratamento de efluentes.
\end{abstract}

\begin{abstract}
Among advanced effluent treatment technologies, membrane separation processes stand out for their wide range of applications, which water desalination, industry process, and water treatment. The nanoparticles used as additives in the membrane formation can bring improvements to the performance and effectiveness of membrane filtration, thus forming nanocomposite membranes, which, depending on their matrix, may have desirable and undesirable characteristics. In most cases, polyamide membranes have been widely studied and used as well in their pure form as with the addition of one or more additives, which can confer important improvements, such as reducing roughness, increasing porosity, correcting defects, improving permeability, among others. Titanium dioxide $\left(\mathrm{TiO}_{2}\right)$ is one of the additives that has achieved prominence as an option to increase porosity, permeability, hydrophilicity, and reduce the effect of fouling. As such, it is applied in several experimental studies that describe their behavior when it is incorporated into membranes. This study aims to review the main cases involving $\mathrm{TiO}_{2}$ used as an additive in polymeric membranes and to present the effects caused by, according to the methods and techniques used in recent literature.
\end{abstract}

Keywords: Polymeric membranes; Inorganic compound; Hybrids; Wastewater treatment. 


\begin{abstract}
Resumen
Entre las tecnologías avanzadas de tratamiento de efluentes, los procesos de separación por membranas destacan por su amplia gama de aplicaciones. Las nanopartículas utilizadas como aditivos en la formación de membranas pueden aportar mejoras en el rendimiento y la eficacia de las membranas como medio filtrante, formando así membranas nanocompuestas que, dependiendo de su matriz, pueden tener características deseables e indeseables. En la mayoría de los casos, las membranas de poliamida han sido ampliamente estudiadas y utilizadas tanto en su forma pura como con la adición de uno o más aditivos, los cuales pueden conferir mejoras importantes, como reducir la rugosidad, aumentar la porosidad, corregir defectos, mejorar la permeabilidad, entre otros. El dióxido de titanio $\left(\mathrm{TiO}_{2}\right)$ es uno de los aditivos que ha alcanzado prominencia como opción para aumentar la porosidad, permeabilidad, hidrofilicidad y reducir el efecto de incrustaciones. Como tal, se aplica en varios estudios experimentales que describen su comportamiento cuando se incorpora a membranas. Este estudio tiene como objetivo revisar los principales casos de $\mathrm{TiO}_{2}$ utilizado como aditivo en membranas poliméricas y presentar los efectos provocados por, de acuerdo con los métodos y técnicas utilizados en la literatura reciente.
\end{abstract}

Palabras clave: Membranas poliméricas; Compuesto inorgânico; Híbridos; Tratamiento de efluentes.

\title{
1. Introdução
}

As tecnologias avançadas que estão geralmente em uso para o tratamento de águas e efluentes são adsorção, extração e filtração por membranas. Estas envolvem, usualmente, a remoção de poluentes orgânicos por meio de transferência de massa e, por isso, são mais adequadas como tratamentos complementares aos métodos tradicionais nas etapas de pré-tratamento ou pós, especialmente para águas residuais industriais (De Gisi et al., 2016).

A tecnologia envolvida nos processos de separação por membranas (PSM) emprega a simulação de uma membrana natural por meio de um material sintético, aplicando as suas características físicas de permeabilidade e seletividade para retenção de partículas indesejáveis durante uma filtração com uma força exterior. A separação por membranas pode ser realizada pelos processos de: destilação, diálise, eletrodiálise, microfiltração, nanofiltração, osmose inversa, pervaporação, separação de gases e ultrafiltração (Galvão \& Gomes, 2015). As vantagens principais dos PSM são alta eficácia, facilidade de operação, tamanho diminuto e consumo reduzido de energia (Babu, O’Connor \& Seeram, 2013).

As membranas sintéticas são preparadas a partir de duas classes distintas de materiais: os orgânicos e os inorgânicos. As membranas inorgânicas apresentam maior vida útil do que as membranas orgânicas. Entretanto, em virtude da maior versatilidade em se obter diferentes morfologias e de apresentarem menor custo, as membranas poliméricas (orgânicas) são as mais utilizadas, apresentando perspectivas significativas de crescimento em termos mercadológicos (Mulder, 1996; Baker, 2004).

Os polímeros sintéticos mais utilizados na preparação de membranas são as poliamidas (náilons), polisulfonas, poliacrilonitrilas, policarbonatos, polieterimidas, poli (fluoreto de vinilideno), entre outros. Essas membranas apresentam não só melhor resistência química como também resistência térmica. Além de boa resistência a compostos clorados, apresentam baixa resistência à compactação mecânica e, ainda, podem ser utilizadas com solventes não-aquosos (Anadão, 2010). As poliamidas 6 e 66 são as mais utilizadas nos setores industriais, principalmente a têxtil. Sua aplicação em nanocompósitos (NCs) se deve as suas propriedades como recuperação elástica, resistência à abrasão e à ruptura, em níveis considerados ótimos (Deopura, 2008; Paz et al., 2008).

As membranas poliméricas podem ser produzidas pela combinação de aditivos e técnicas tais como inversão de fases, sinterização e polimerização, através da formação de camadas de poliamidas de mínima espessura com poliamidas porosas servindo de suporte. As desvantagens inerentes aos PSM, como por exemplo, baixa permeabilidade, podem ser contornadas pela utilização de membranas de nanocompósitos obtidas a partir de prata, zinco, grafeno, de dióxido de titânio $\left(\mathrm{TiO}_{2}\right)$, de compostos inorgânicos como argilas, dentre outros (Susuki et al., 2016; Shen et al., 2018). Sendo assim, o objetivo deste estudo é realizar um levantamento bibliográfico sobre a aplicação do dióxido de titânio $\left(\mathrm{TiO}_{2}\right)$ como aditivo na forma de nanopartícula (NP) para a melhoria do desempenho de membranas híbridas a partir de poliamidas. 


\section{Metodologia}

Para a produção deste estudo, uma revisão bibliográfica qualitativa, buscou realizar uma pesquisa sistemática nas bases de dados SciELO, Sciencedirect e Web of Science, que contemplasse artigos de períodicos com o assunto de interesse, de preferência por artigos da última década. Os resultados da pesquisa foram obtidos durante todo o primeiro semestre de 2021, sendo as palavras chaves utilizadas: "Membranes", " $\mathrm{TiO}_{2}$ ", "Polyamides", "Additive", "Nanoparticle", "Polymer" e "Nanocomposites", com limitação temporal entre o período de 2003 a 2021. O refinamento ocorreu mediante avaliação dos autores, sendo obrigatória a correlação entre os termos membranas, polímeros e $\mathrm{TiO}_{2}$ para adição do estudo publicado nesta pesquisa, dado que o objeto de interesse principal é a demonstração da utilização do dióxido de titânio como aditivo para melhoria das características de membranas com base polimérica, associado ou não a outros aditivos.

\section{Resultados e Discussão}

\subsection{Membranas e seus Processos de Separação}

Membranas são estruturas sólidas ou líquidas que atuam como delimitadores de espaços e como filtros, que visam reter partículas e permitir a passagem de outras, cujo diâmetro seja menor do que o de seus poros (Figura 1). De acordo com a sua natureza química, as membranas podem ser biológicas ou artificiais (sintéticas). As membranas sintéticas, produzidas em laboratório, são classificadas em orgânicas e inorgânicas. A propriedade das membranas que permite o fracionamento de moléculas e a separação de substâncias como sendo um meio filtrante é a porosidade (Matsuura, 1993).

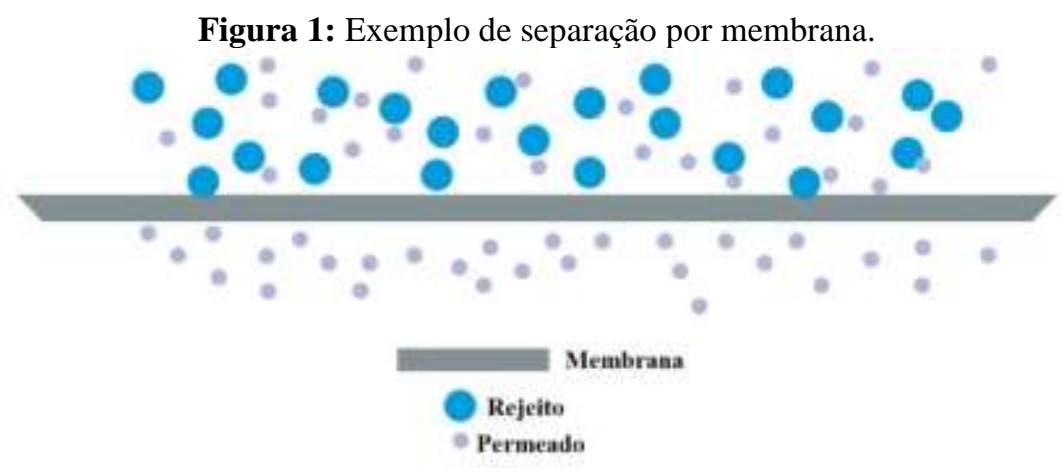

Fonte: Adaptado de Kasvi (2017).

Em sua maioria, para produzir membranas, utiliza-se materiais poliméricos (orgânicos) e cerâmicos (inorgânicos) com a seguinte formação sequencial: suporte poroso com uma fina camada superficial. O suporte poroso oferece estabilidade mecânica para a membrana, enquanto que a camada superior promove a permeabilidade necessária. A membrana ideal possui uma estrutura forte, resistência às mudanças de temperatura, a desgastes químicos, como alterações no pH e à incrustações, distribuição correta dos poros e alta rejeição de partículas. Para tanto, deve-se configurar a morfologia da membrana, sua geometria, montagem e orientação relativa ao fluxo de água (Judd, 2010).

A fim de se obter uma melhor compreensão sobre a morfologia das membranas, utiliza-se procedimentos que empregam imagens com resolução suficiente para observar características da membrana, caracterizações por microscopias, como a de Força Atômica (MFA), Eletrônica de Varredura (MEV) e MEV por Emissão de Campo (Deowan et al., 2015).

A filtração é um processo que permite eliminar impurezas que tendem a se acumular em algum meio, seja ele líquido ou gasoso. Entre os meio filtrantes mais utilizados estão as membranas, classificadas em membranas de microfiltração (MF), ultrafiltração (UF), nanofiltração (NF) e osmose inversa (OI), apresentados na Figura 2 (Liderfelt \& Royce, 2017). 

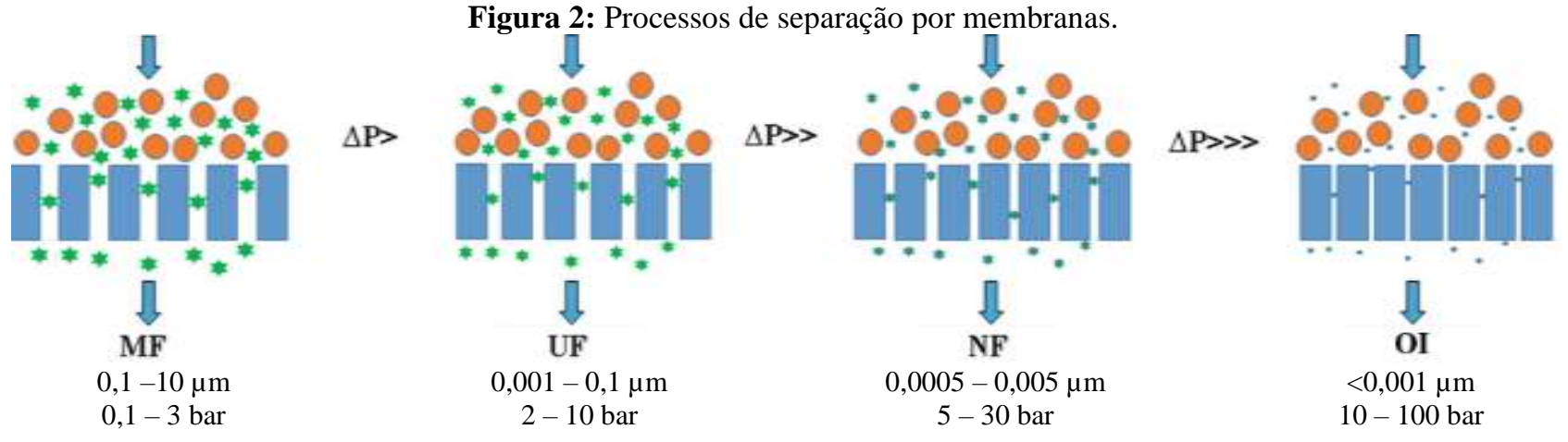

Fonte: Adaptado de Singh et al. (2020).

A microfiltração (MF) é um processo realizado ao longo de uma membrana microporosa induzido por pressão para reter partículas contaminantes com um alcance na ordem 0,1-10 $\mu \mathrm{m}$, como cianobactérias, protozoários e endotoxinas. Associar a microfiltração com outras técnicas, por exemplo, utilizar sequencialmente a osmose inversa, aumenta o desempenho do sistema (Pham \& Dang, 2019).

A ultrafiltração (UF) é um método de separação de impurezas como vírus e bactérias, por meio de uma pressão externa de média intensidade. As aberturas de poros variam de 0,01-0,1 $\mu \mathrm{m}$. É considerado um método limpo por não produzir resíduos químicos e um efluente de alta qualidade. Quando a membrana é utilizada, busca-se aumentar a sua hidrofilicidade, para reduzir as possibilidades de incrustações (Cordier et al., 2020; Yong et al., 2019).

A nanofiltração (NF) é utilizada nos processos de extração de produtos vegetais em solução alcoólicas e aquosas. As aberturas de poros variam de 0,001-0,01 $\mu \mathrm{m}$. A separação é obtida através da membrana pela aplicação de um gradiente de pressão. Esta tecnologia é usada na separação de substâncias orgânicas, tais como micropoluentes e íons polivalentes (Aquino, 2011).

A osmose inversa (OI) é uma filtração por membranas de íons monovalentes e aminoácidos de soluções à base d'água utilizando um gradiente de concentração hidrostático, tendo aberturas de poros inferiores a $1 \mathrm{~nm}$. Nesta técnica, deve-se considerar a permeabilidade e a rejeição de sal das membranas e a resistência geral à incrustação. De forma a melhorar estas características com tecnologias, se faz necessárias alterações na superfície das membranas e a utilização de aditivos (Arribas $e t$ al., 2015; Lilane et al., 2020).

\subsection{Matriz Utilizada na Obtenção das Membranas Poliméricas}

Polímero é uma palavra que tem sua origem no idioma grego e significa muitas partes, e, por esta razão, representa moléculas químicas extensas, em que seu formato depende de seu tamanho, pertencentes a um grupo de materiais sintetizados ou extraídos de produtos naturais, com certas características como leveza e maleabilidade, utilizadas usualmente como matriz (material base) na formação de membranas (Carraher Jr., 2012; Teegarden, 2004).

A poliamida foi criada no ano de 1934, a partir dos estudos sobre fibras de poliésteres, e observou-se um tipo com alto ponto de fusão com boas propriedades elásticas, o polímero de aminoetiléster. As poliamidas fazem parte do grupo de polímeros cristalinos que são tipicamente formados por meio do processo de condensação de um diácido e uma diamina (McKeen, 2019).

A poliamida 6 (conhecida comercialmente por náilon 6), cuja estrutura química pode ser observada na Figura 3, possui o complemento seis para caracterizar o número de átomos de carbono no monômero. Dentre as suas propriedades, destacam-se a sua superfície mais lisa, boa absorção e aderência à umidade, resistência ao desgaste, a produtos químicos, ao calor e à abrasão, impermeabilidade à gasolina, óleos e graxas. Para a sua criação, é necessária a utilização de catalisadores, 
como a água, na reação de policondensação do ácido aminocapróico, também chamado de caprolactama (Colombi, 2016; Wypych, 2016).

Figura 3: Estrutura química da poliamida $6-\left(\mathrm{C}_{6} \mathrm{H}_{11} \mathrm{NO}\right)_{\mathrm{n}}$.

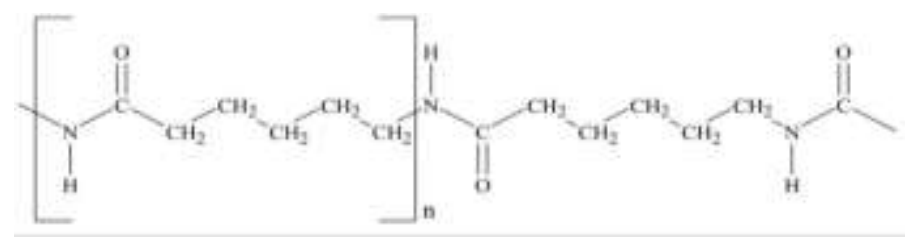

Fonte: McKeen (2019).

A poliamida 6,6 (conhecida comercialmente por náilon 66), apresentada na Figura 4, é uma fibra polimérica insolúvel, possui alto ponto de fusão, rigidez e elasticidade, é resistente ao desgaste, à abrasão, à umidade e ao calor, e apresenta características superiores à seda, que é o seu equivalente natural. Ela é sintetizada a partir da condensação das matérias-primas 1,6-hexametilenodiamina (6 carbonos) e ácido adípico (6 carbonos), por isso é chamada de poliamida 6,6, enquanto que a caprolactama, matéria-prima da poliamida 6 possui apenas 6 carbonos no monômero. A poliamida 6 e a 6,6 possuem como característica similar em sua estrutura química molecular a presença de ligações de hidrogênio com grupo amida $(\mathrm{C}=\mathrm{O}-\mathrm{N})$ (Carraher Jr., 2012).

Figura 4: Estrutura química da poliamida $6,6-\left(\mathrm{C}_{12} \mathrm{H}_{22} \mathrm{~N}_{2} \mathrm{O}_{2}\right)_{\mathrm{n}}$.

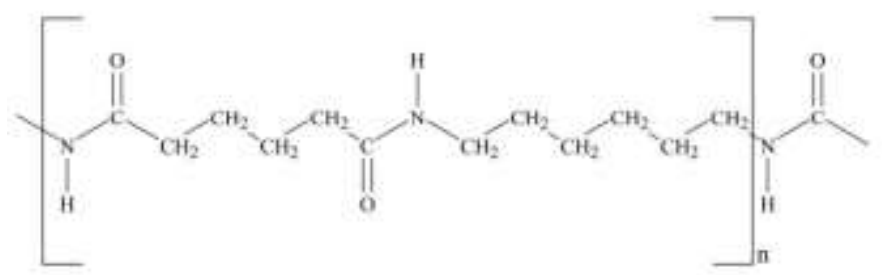

Fonte: McKeen (2019).

\subsection{Aditivo utilizado na produção de membranas com $\mathrm{TiO}_{2}$}

O dióxido de titânio $\left(\mathrm{TiO}_{2}\right)$ é um óxido metálico, um minério purificado quimicamente, e que pode ser sintetizado como nanotubos e nanofibras, por exemplo. Possui propriedades físicas, químicas e ópticas relativas à sua forma natural, que inclui seus três estados polimórficos cristalinos: anatase (tetragonal, densidade de 3,89 g. $\mathrm{cm}^{-3}$ ), rutilo (tetragonal, densidade de $4,25 \mathrm{~g} . \mathrm{cm}^{-3}$ ) e broquita (ortorrômbica, densidade de 4,12 g.cm ${ }^{-3}$ ) (Zhao et al., 2009).

A síntese das nanopartículas de $\mathrm{TiO}_{2}$, que são utilizadas na obtenção de membranas híbridas, pode ser realizada das seguintes formas: técnica hidrotérmica, sólido-gel, deposição térmica, química em fase de vapor, reação de combustão, oxidação, eletrodeposição e micela inversa (Akakuru et al., 2020; Ribeiro et al., 2010). A anatase é normalmente produzida em síntese pelo método sol-gel, mas a broquita é frequentemente observada como subproduto quando a hidrólise do precursor, seguida de precipitação, é realizada em meio ácido e a baixa temperatura (Mutuma et al., 2015). A Figura 5 ilustra as estruturas dos polimorfos cristalinos anatase, rutilo e broquita do $\mathrm{TiO}_{2}$. 
Figura 5: Estruturas dos polimorfos cristalinos do $\mathrm{TiO}_{2}$.

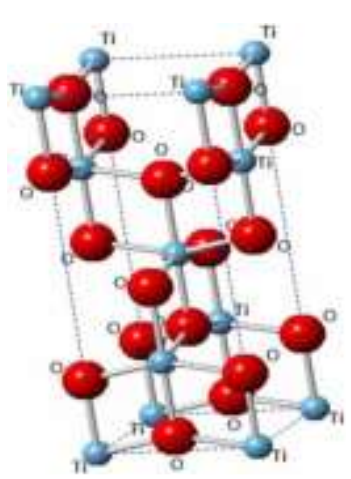

Anatase

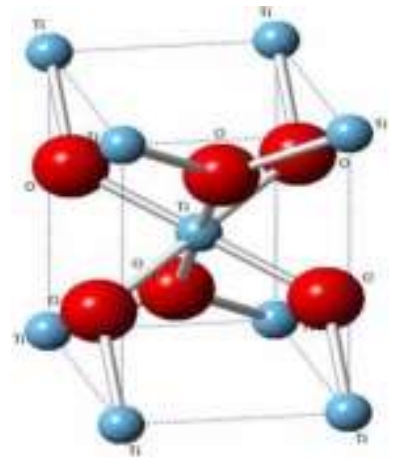

Rutilo Fonte: Santos (2017)

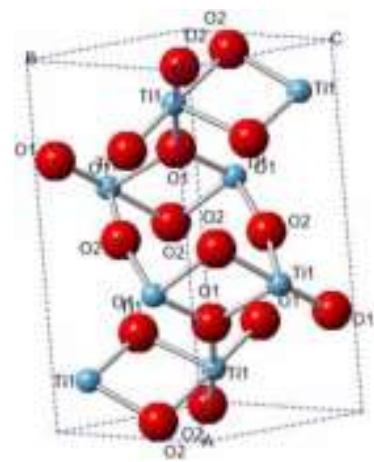

Broquita

As nanopartículas de $\mathrm{TiO}_{2}$ são aplicáveis em diversas áreas, como na energia solar durante a fabricação de placas fotovoltaicas, técnicas de tratamento avançadas de efluentes, melhoria da qualidade do ar, em indústrias de alimentos, de cosméticos e de tinturas, na descontaminação de solos, dentre outras (Bai et al., 2014). Suas principais propriedades são: excelente estabilização química em meio aquoso e mecânico, resistência à corrosão, melhoria na hidrofilicidade de membranas poliméricas, e sua produção é considerada de baixo custo (Li et al., 2007; Grimes e Mor, 2009).

\subsection{Utilização do $\mathrm{TiO}_{2}$ em Membranas Híbridas}

Kim et al. (2003) desenvolveram membranas de poliamidas aromáticas à base de nanopartículas de $\mathrm{TiO}_{2}$ para reduzir a ocorrência da bioincrustação microbiana. Essas membranas foram incorporadas a membranas comerciais a base de polisulfona (PSU) e tecido-nãotecido (TNT), formando uma membrana híbrida, conforme a Figura 6. As nanopartículas de $\mathrm{TiO}_{2}$ foram fabricadas e caracterizadas por Difração de Raios-X (DRX) e Microscopia Eletrônica de Transmissão (MET). Além disso, as membranas híbridas foram caracterizadas por Espectroscopia de Fotoelétrons de Raios-X (XPS) e fluxo para analisar a eficiência. Por DRX e Microscopia Eletrônica de Varredura (MEV) foram constatados elementos de tamanho de partículas característicos do $\mathrm{TiO}_{2}$.

Através da análise XPS, determinou-se a formação de uma película fina de nanocompósitos de $\mathrm{TiO}_{2}$. Verificaram-se os efeitos fotobactericidas destas membranas híbridas nos coliformes E. coli sob e sem análise espectral de Ultravioleta (UV), resultando em maiores eficiências sob a influência da radiação, possuindo os maiores valores do fluxo de transporte de água e rejeição de sal. Também verificaram que a membrana híbrida possui efeitos preventivos à incrustações microbiológicas por meio da osmose inversa (OI), pois as espécies antibacterianas produzidas pela membrana híbrida matam as células bacterianas e evitam a fixação da bactéria à superfície da membrana.

Figura 6: Desenho esquemático da membrana híbrida.

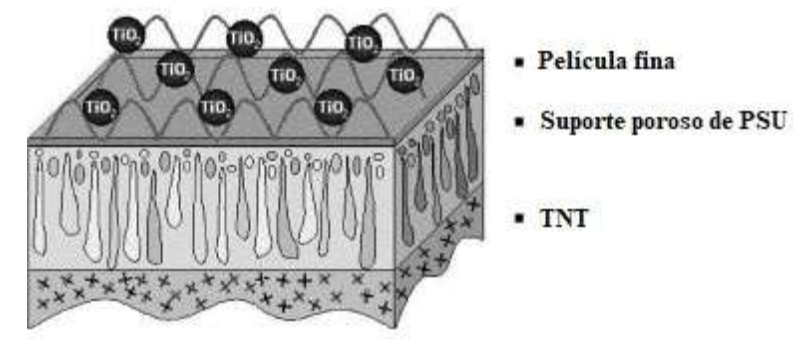

Fonte: Adaptado de Kim et al. (2003). 
Lee et al. (2008) obtiveram membranas de nanocompósitos de poliamida (PA) com nanopartículas de $\mathrm{TiO}_{2}$, preparadas a partir de um suporte de polietersulfona (PES), revestido com solução aquosa de m-fenil diamina (MPD) e em seguida imerso em solução aquosa de cloreto de trimesoila orgânico (TMC) e nanopartículas de $\mathrm{TiO}_{2}$ produzindo uma polimerização interfacial, que resultou em uma membrana contendo uma camada de filme fino de $\mathrm{PA} / \mathrm{TiO}_{2}$. $\mathrm{As}$ membranas foram caracterizadas por: MEV por emissão de campo, DRX, Espectroscopia de Infravermelho com Transformada de Fourier (FTIR) e XPS. A partir do MEV foi constatado uma dispersão homogênea das nanopartículas que gerou uma estabilidade nas propriedades mecânicas das membranas. Da análise de XPS concluiu-se que quantidades expressivas do $\mathrm{TiO}_{2}$ permaneceram na superfície das membranas. Em relação ao desempenho da membrana, o tempo de cura adequado foi de 5 minutos numa temperatura de $70{ }^{\circ} \mathrm{C}$ com retenção de sulfato de magnésio $\left(\mathrm{MgSO}_{4}\right)$ acima de $95 \%$. As membranas são hidrofílicas, propriedade constatada pelo fluxo do permeado, que foi de 9,1 L. m-2.h-1 a uma pressão de 0,6 MPa, um valor elevado devido à maior quantidade de $\mathrm{TiO}_{2}$ aderidas na poliamida. A concentração adequada de $\mathrm{TiO}_{2}$ nas membranas foi de $5 \%$ em peso. A membrana produzida e seu processo de preparação estão apresentados na Figura 7.

Figura 7: Esquema de preparação das membranas $\mathrm{PA} / \mathrm{TiO}_{2}$.
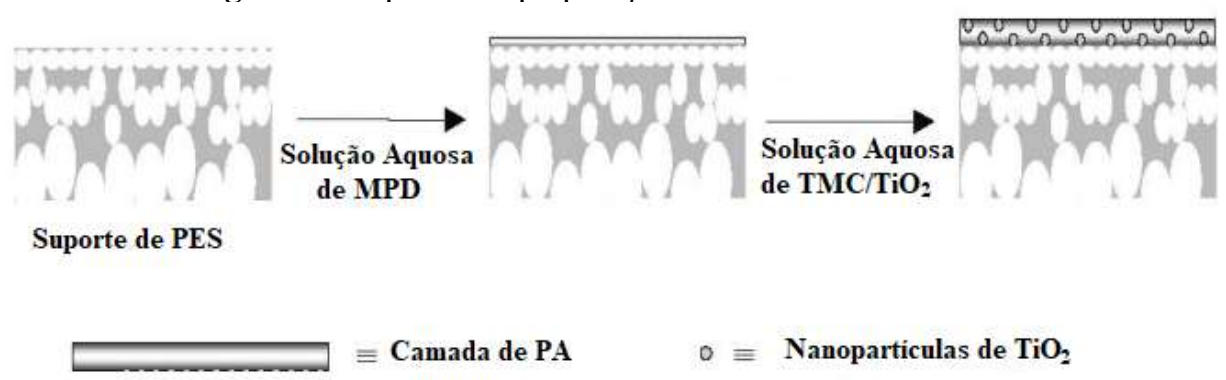

Fonte: Adaptado de Lee et al. (2008).

Rajaeian et al. (2013) prepararam membranas de nanocompósitos de filme fino de poliamida (TFN) para o processo de nanofiltração via incorporação interfacial de nanopartículas de $\mathrm{TiO}_{2}$, em sua forma pura e funcionalizado na solução orgânica cloreto de trimesoílo (TMC). A Figura 8 mostra a diferença entre o arranjo das nanopartículas do $\mathrm{TiO}_{2}$ (a) sem a dispersão de TMC e (b) com a dispersão de TMC. Com a modificação, observou-se que as nanopartículas são homogeneamente dispersas, reduzindo a formação de aglomerados. A funcionalização da superfície do $\mathrm{TiO}_{2}$ foi confirmada por DRX, FTIR e análise espectral de UV visível. As propriedades de superfície das membranas compostas fabricadas foram investigadas usando MEV, Espectroscopia por Dispersão de Energia (EDS), Microscopia de Força Atômica (MFA) e Ângulo de Contato (AC). $\mathrm{O} \mathrm{TiO} 2$ funcionalizado em baixas concentrações melhorou a rejeição de sal para 54\%, bem como o fluxo de água para 12,3 L.m-2.h-1. Ao incorporar uma maior concentração de $\mathrm{TiO}_{2}$, o fluxo de água aumentou até 2 vezes em comparação com a membrana de poliamida pura com pequena perda de rejeição.

Figura 8: Esquema de preparação das membranas $\mathrm{PA} / \mathrm{TiO}_{2}$.

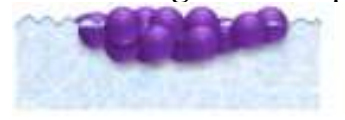

a

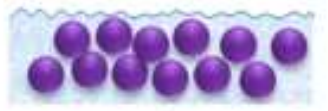

b

\section{Pelicula fina de poliamida Nanoparticulas de $\mathrm{TiO}_{2}$}

Fonte: Adaptado de Rajaeian et al. (2013).

Anan et al. (2015) preparam uma membrana fotocatalítica de filme fino de poliamida incorporada para remoção de bisfenol A (BPA), composto considerado um desregulador endócrino por possuir propriedades estrogênicas, adicionando TiO 2 . 
A membrana TFC foi preparada a partir de M-fenilenodiamina e cloreto de trimesoíla por meio da técnica de polimerização interfacial. As concentrações do $\mathrm{TiO}_{2}$ foram de $0,1 \%, 0,2 \%, 0,3 \%, 0,4 \%$ e $0,5 \%$ em peso. As membranas produzidas foram caracterizadas por MEV por emissão de campo, Espectroscopia de energia dispersiva de raios-X (EDX), FTIR e AC. Para avaliar a eficiência da membrana, foram utilizadas águas residuais farmacêuticas sintéticas contendo BPA para serem tratadas em um reator fotocatalítico. Os resultados das caracterizações indicaram que as propriedades das membranas melhoraram em relação ao aumento em peso de $\mathrm{TiO}_{2}$. Todas as membranas apresentaram mais de $90 \%$ de rejeição de $\mathrm{BPA}$, sendo a maior rejeição $99,9 \%$ pertencente a concentração de $0,4 \%$ em peso de $\mathrm{TiO}_{2}$. Assim, membranas fotocatalíticas híbridas de $\mathrm{TFC} / \mathrm{TiO}{ }_{2}$ são potencialmente eficazes na remoção do composto fármaco BPA.

Tayefeh et al. (2015) analisaram membranas de TFN de poliamida por OI, a partir de nanopartículas de magnetita $\left(\mathrm{Fe}_{3} \mathrm{O}_{4}\right)$ e $\mathrm{TiO}_{2}$ adicionadas por polimerização interfacial ou por colóide em TMC ou em MPD. Estudou-se o efeito do revestimento utilizado na sua camada, bem como as análises de MEV, FTIR com Reflexão Total Atenuada (ATR) e AC. O maior percentual das nanopartículas diminuiu a rugosidade da superfície, favorecendo a hidrofilicidade das membranas. A adição das nanopartículas de $\mathrm{Fe}_{3} \mathrm{O}_{4}$ e $\mathrm{TiO}_{2}$ na solução MPD, resultou em um maior controle na dispersão e aglomeração destas partículas na superfície das membranas.

Ngo et al. (2016) estudaram o efeito no desempenho e nas propriedades das membranas de poliamida TFC a partir da adição de nanopartículas de $\mathrm{TiO}_{2}$ em sua superfície, com irradiação UV posterior, caracterizando eventuais alterações. O desempenho das membranas revestidas com $\mathrm{TiO}_{2}$ em separar corantes em uma solução aquosa foi superior ao ser comparado ao desempenho das membranas sem revestimento. Ao aumentar as taxas de fluxo, pôde-se observar a melhoria da propriedade anti-incrustante. Além disso, a presença de revestimento aumentou a hidrofilicidade da superfície da membrana, comprovada através análises do ângulo de contato com a água.

Dinari e Haghighi (2017) após modificarem a superfície de nanopartículas de $\mathrm{TiO}_{2}$ com o agente de acoplamento 3aminopropiltrietoxissilano (APTES), introduzido por irradiação ultrassônica, preparam membranas híbridas de poliamida com 5, 10 e $15 \%$ de $\mathrm{TiO}_{2}$. Para este propósito, prepararam e caracterizaram a superfície dos nanocompósitos de $\mathrm{TiO}_{2}$, bem como da membrana de poliamida/ $\mathrm{TiO}_{2}$-modificado. A membrana PA pura e seus nanocompósitos foram caracterizados por FTIR, DRX e MEV por emissão de campo e Microscopia Eletrônica de Transmissão (MET). As análises de microscopia provaram que o APTES foi enxertado na superfície do $\mathrm{TiO}_{2}$, que o $\mathrm{TiO}_{2}$ estava bem disperso na matriz polimérica, sem aglomerados, com o aumento progressivo da porcentagem. Cada uma das porcentagens dos nanocompósitos de $\mathrm{TiO}_{2}$ teve um rendimento progressivo para remoção do cromo (VI), variando entre $76,25 \%$ a $86,25 \%$, comprovando que as membranas com $\mathrm{TiO}_{2}$ modificado são bons adsorventes. A Figura 9 apresenta as mudanças na estrutura das nanopartículas de $\mathrm{TiO}_{2}$ após receber as modificações antes de serem introduzidos nas membranas.

Figura 9: Estrutura do $\mathrm{TiO}_{2}$ após a modificação com o agente de acoplamento APTES

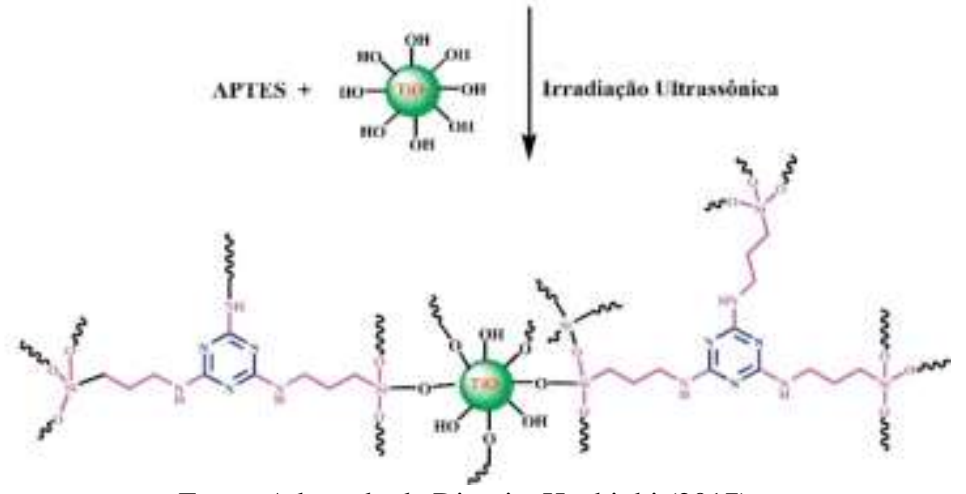

Fonte: Adaptado de Dinari e Haghighi (2017). 
Shao et al. (2017) estudaram o processo de OI utilizando membranas modificadas com nanopartículas de $\mathrm{TiO}_{2}$ e de óxido de grafeno (OG), montadas em camadas na superfície das membranas, para aumentar a resistência ao cloro e a bioincrustação. Realizou-se análises de AC e XPS que resultaram em melhorias em relação à hidrofilicidade das superfícies das membranas e mostraram que os dois materiais estavam ligados as superfícies das membranas em condições estáveis. O OG contribuiu para desenvolver resistência ao cloro e o $\mathrm{TiO}_{2}$ trouxe as membranas um efeito anti-incrustante. As membranas modificadas exibiram melhorias no fluxo do permeado, em relação ao fluxo das membranas puras. Além disso, membranas revestidas em camadas foram $75 \%$ mais eficazes em resistir ao cloro do que as sem revestimentos para o mesmo período de 20 $\mathrm{h}$ de exposição.

Isawi (2018) preparou membranas de nanocompósitos de poliamida TFC com os aditivos $\mathrm{ZnO} / \mathrm{TiO}_{2}$ associados ao (poli) ácido metacrílico (PMAA), um composto que possui solubilidade em água e biocompatibilidade com os demais materiais, para serem utilizadas na remoção de sal da água. As membranas foram construídas em camadas e os nanocompósitos sintetizados foram enxertados na camada ativa da membrana de PA, sobre uma camada de suporte microporoso de PSU, resultando em membranas de ( $\mathrm{ZnO} / \mathrm{TiO}_{2} / \mathrm{PMAA}$ )-g-PA(TFC), com distribuição uniforme dos NCs na superfície da membrana, altamente permeável, conforme Figura 10. Elas foram caracterizadas por FTIR, DRX, MEV e AC e avaliadas quanto ao seu desempenho na separação dos sais. A caracterizações indicaram que os nanocompósitos aumentaram a cristalinidade e as forças intermoleculares que operam na membrana em comparação com a membrana pura, tornando-a mais resistente mecanicamente, trouxeram alta hidrofilicidade da membrana com o aumento da concentração de NCs. A retenção dos sais da solução de $\mathrm{NaCl}$ de 2000 mg.L-1 foi de 96,8\% e o fluxo de água de 33 L.m-2.h-1 numa pressão de 15 bar. Foi constatado que as membranas de NCs melhoraram o desempenho da membrana de PA, em relação as suas propriedades mecânicas, resistência à incrustação (testada com albumina de soro bovino) e a bioincrustação (testada com E. coli), hidrofilicidade em termos de ângulo de contato e estabilidade em soluções ácido-alcalinas.

Figura 10: Estrutura química da membrana híbrida ( $\left.\mathrm{ZnO} / \mathrm{TiO}_{2} / \mathrm{PMAA}\right)$-g-PA(TFC).

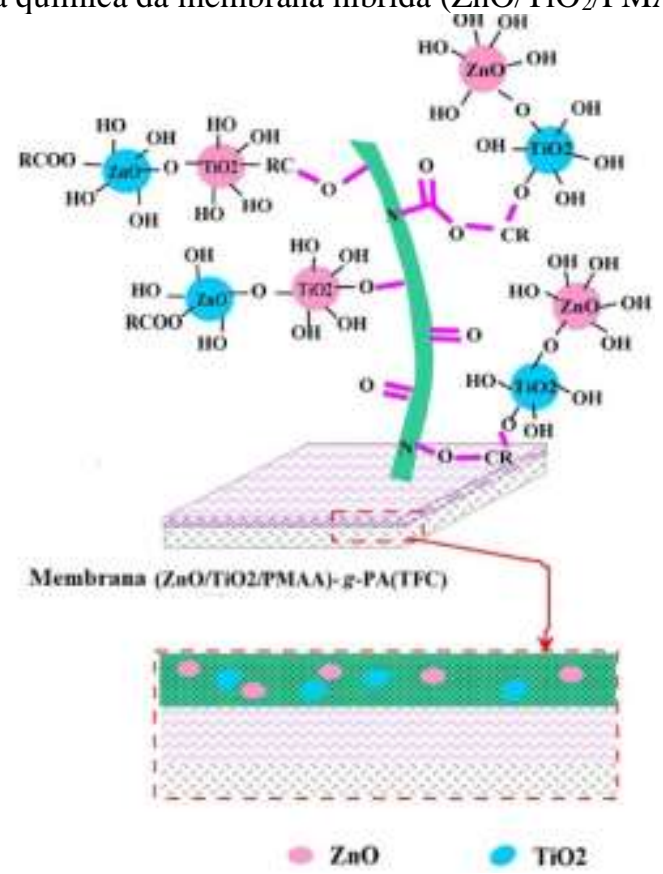

Fonte: Adaptado de Isawi (2018).

Li et al. (2018) desenvolveram membranas de nanocompósitos de poliamida a base de $\mathrm{TiO}_{2}$ por meio da técnica de polimerização interfacial, para nanofiltração (NF) de sais da água. A fim de caracterizar morfologicamente as membranas, realizaram-se as análises de MEV, MFA, XPS, AC e estudos dos efeitos da concentração de monômero, o tempo de reação e 
de imersão na fase aquosa. As membranas atingiram um fluxo de água de $105 \mathrm{~L} \cdot \mathrm{m}^{-2} \cdot \mathrm{h}^{-1}$, sendo considerado um alto fluxo, retendo cerca de 95,5\% do $\mathrm{MgCl}_{2}$ (sal testado) a um pressão de 4 bar. Constatou-se que as suas propriedades anti-incrustantes, estabilidade a longo prazo e a recuperação do permeado foram notáveis, graças ao $\mathrm{TiO}_{2}$, que promoveu resistência à incrustação e melhoria da molhabilidade da membrana, além do fato da membrana ser superhidrofílica, a tornam ideal para ser aplicada no tratamento e dessalinização de águas.

Baig et al. (2019) utilizaram nanocompósitos em um suporte poroso de PSU capazes de transportar vapor de água com alta permeabilidade. Esses materiais foram o $\mathrm{OG}$ e o $\mathrm{TiO}_{2}$, incorporados de forma conjunta e separadamente a membranas TFC de PA, preparadas por polimerização interfacial, variando a concentração entre 0,05 a 2,0 \% em peso de cada aditivo. As membranas receberam o revestimento dos NCs e banhos consecutivos em soluções contendo MPD e TCM, resultando em membranas TFN de $\mathrm{OG}$ e de $\mathrm{OG} / \mathrm{TiO}_{2}$, que foram analisadas química e fisicamente, por FTIR de refletância total atenuada (ATR-FTIR), EDX, Termogravimetria (TGA), MET, MEV, MFA, AC e XPS. Dentre os resultados, encontrou-se que ambos os nanomateriais se aderiram ao longo da superfície da poliamida mediante ligações covalentes e de hidrogênio. As membranas de nanocompósitos a base de $\mathrm{OG}-\mathrm{TiO}_{2}$ obtiveram resultados superiores para a rugosidade de superfície, hidrofilicidade, possuíram uma maior quantidade de grupos carboxila e menor grau de reticulação, apresentando maior permeabilidade em comparação com as membranas de nanocompósitos a base apenas de OG. A Figura 11 apresenta as membranas híbridas contendo óxido de grafeno produzidas com e sem adição de $\mathrm{TiO}_{2}$.

Figura 11: Membranas TFN/OG e TFN/OG- $\mathrm{TiO}_{2}$ produzidas por polimerização interfacial.

OG $\mathrm{OG}-\mathrm{TiO}_{2}$

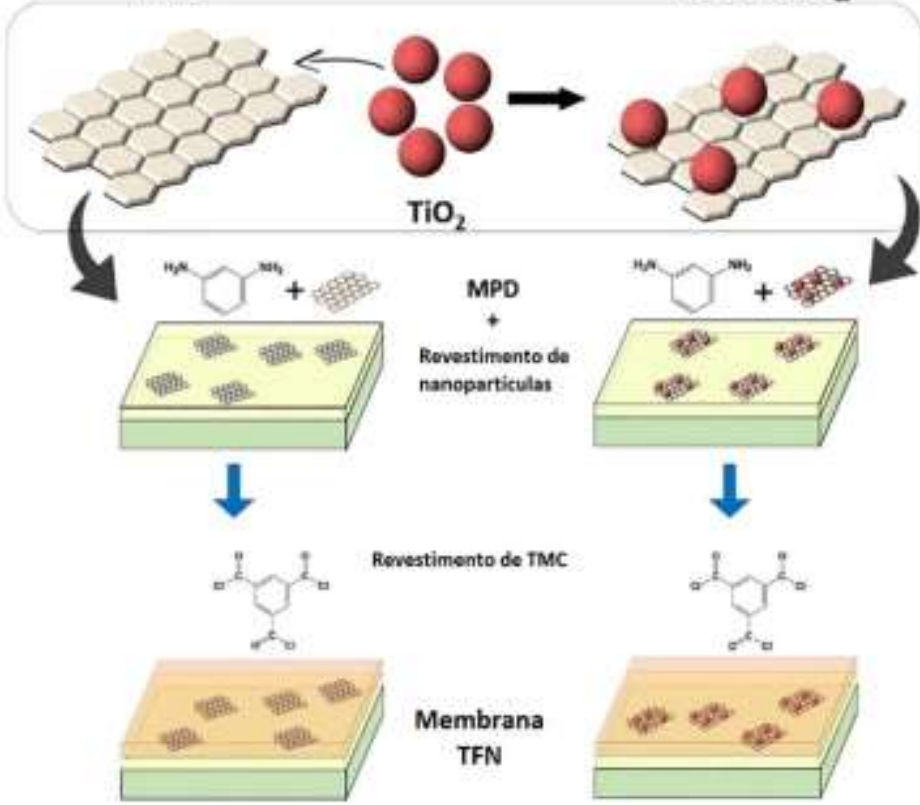

Fonte: Adaptado de Baig et al. (2019).

Liu et al. (2019) buscaram melhorar a performance da propriedade Pervaporação (PV) produzindo membranas de compósitos de poliamida com película fina TFC através de polimerização interfacial, utilizando o substrato da membrana cerâmica de fibra oca $\alpha-\mathrm{Al}_{2} \mathrm{O}_{3}$ (CHF), modificadas com nanopartículas de $\mathrm{TiO}_{2}$ em sua superfície. Este substrato foi preparado a partir da fibra oca com a fase termodinamicamente estável do óxido de alumínio $\left(\alpha-\mathrm{Al}_{2} \mathrm{O}_{3}\right)$ para a formação de uma camada de poliamida. Esta camada resultante é rugosa e macroporosa, portanto, para preencher os macroporos e melhorar a lisura, tornando a superfície sem defeitos, houve o revestimento do CHF com $\mathrm{TiO}_{2}$ pelo método sol-gel com o cobre (VI), criando uma camada intermediária, tornando a superfície da membrana de rugosa para lisa, como demonstrado pela Figura 12. 
Foram realizadas análises de AC, MEV por emissão de campo, MFA, XPS e de pervaporação. As caracterizações indicaram que houve a formação de uma camada intermediária fina, e com isso, a membrana se tornou menos rugosa e mais hidrofílica, pois o $\mathrm{TiO}_{2}$ e o substrato de cerâmica são materiais hidrofílicos. Em relação a pervaporação, o melhor desempenho das membranas com $\mathrm{TiO}_{2}$ encontrado foi quando o fluxo do permeado é de $6,44 \mathrm{~kg} \cdot \mathrm{m}^{-2} \cdot \mathrm{h}^{-1}$, com fator de separação acima de 12.000 para desidratação por PV de 90\%, pois nesta condição, mesmo após de 100h de processo, as membranas mantiveram o desempenho de separação elevado para aplicação na desidratação de compostos orgânicos.

Figura 12: Membranas TFC de PA/CHF sem e com a camada intermediária de $\mathrm{TiO}_{2}$.

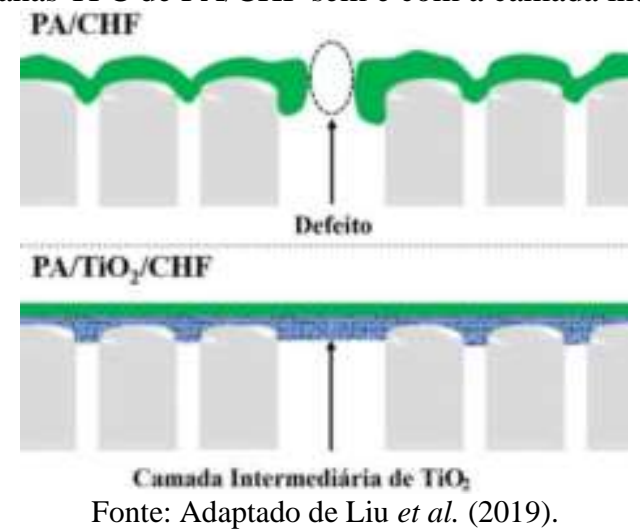

Kedchaikulrat et al. (2020) sintetizaram uma solução coloidal de $\mathrm{TiO}_{2}$ em nanopartículas para gerar membranas de nanocompósitos de filme fino de poliamida (TFN PA) para aplicação no tratamento de água com o mínimo possível de aglomerados das nanopartículas (NPs). A síntese das $\mathrm{NPs}$ de $\mathrm{TiO}_{2}$ ocorreu pelo método de hidrólise (sol-gel) e foram integradas no substrato polimérico poliacrilonitrila (PAN), ao ser realizado o processo de polimerização interfacial para formação da membrana TFN de PA. Estudou-se a membrana por meio de ATR-FTIR, MEV, FMA e AC, bem como por testes de fluxo e incrustração.

Em relação à separação, o desempenho das membranas de nanocompósitos a base de $\mathrm{TiO}_{2}$ em comparação com outras abordagens de produção de $\mathrm{NPs}$ de $\mathrm{TiO}_{2}$, foi considerado um bom desempenho, com melhoria efetiva de 12 a $19 \%$, permeabilidade à água de $2,94 \mathrm{~L} \cdot \mathrm{m}^{-2} \cdot \mathrm{h}^{-1}$ na pressão de 5 bar, rejeição de cloreto de sódio de $94,2 \%$, recuperação do fluxo de até 94\%, propriedade antivegetativa aprimorada e boa resistência à incrustação. Foram essenciais para esses resultados, não só o método de síntese das nanopartículas de $\mathrm{TiO}_{2}$, mas os subprodutos gerados, como ácidos residuais e etanol presentes na membrana, pois impactaram positivamente na molhabilidade, fluxo de água e rejeição de sal. A Figura 13 apresenta a membrana híbrida formada após adição de $\mathrm{TiO}_{2}$.

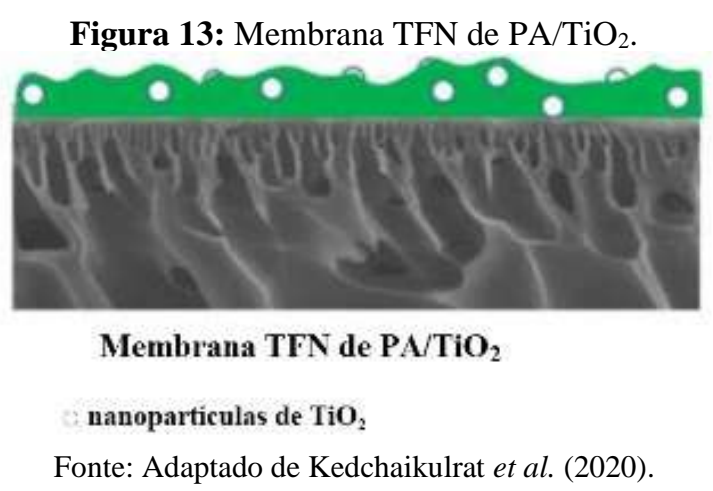

Urper-Bayram et al. (2020) estudaram a adição de nanopartículas em membranas de poliamida e seus efeitos na rejeição de sais, matéria orgânica (MO) e o desempenho do fluxo de permeado nos processos de OI e NF. Produziram 
membranas usando as nanopartículas escolhidas, de $\mathrm{TiO}_{2}$ e $\mathrm{SiO}_{2}$ em quatro concentrações $(0,01,0,05,0,2$ e 0,5\% p/v) cada e membranas sem adição de nanopartículas para controle. Os sais em análise foram cloreto de sódio $(\mathrm{NaCl})$ e sulfato de magnésio $\left(\mathrm{MgSO}_{4}\right)$ e a MO foram os ácidos húmico (HÁ) e tânico (TA). Ambas as membranas com adição de NP, demonstraram resultados melhores em termos da hidrofilia, porosidade e consequente permeabilidade. Os fluxos de permeado de membrana aumentaram com o aumento do teor de $\mathrm{TiO}_{2}$ e $\mathrm{SiO}_{2}$ em até $0,5 \%$ em peso. Para as membranas com $\mathrm{TiO}_{2}$ a permeabilidade foi aumentada em $24 \%$ e as com $\mathrm{SiO}_{2}$ a permeabilidade cresceu $58 \%$, a rejeição $\mathrm{MO}$ foi de pelo menos $77 \%$ para HÁ e 71\% para TA nas membranas com aditivos. As membranas com NPs aumentaram a rejeição de $\mathrm{MgSO}_{4}$ e a rejeição de $\mathrm{NaCl}$, sendo que o desempenho da rejeição de $\mathrm{NaCl}$ foram semelhantes nas quatro concentrações.

Zarshenas et al. (2020) modificaram a interface inferior da membrana de compósito de filme fino (TFC) de poliamida aplicando o nanofilme de $\mathrm{TiO}_{2}$ ao monômero na subcamada atômica depositada (ALD) para realizar o controle da polimerização interfacial durante a fabricação de membranas projetadas para o processo de osmose inversa. A permeabilidade da membrana produzida foi de $1,8 \mathrm{~L} \cdot \mathrm{m}^{-2} \cdot \mathrm{h}^{-1} \cdot \mathrm{bar}^{-1} \mathrm{e}$ a retenção salina de $96,1 \%$, ambos os parâmetros foram considerados altos, tornando essa técnica de otimização de materiais (revestimento da subcamada ALD de nanomateriais) utilizável para melhorar os processos de tratamento de água e dessalinização, desde que com um número limitado de até 200 de ciclos ALD, para não prejudicar o bom desempenho da membrana TFC.

Al-Gamal, Falath e Saleh (2021) avaliaram a eficiência das membranas de poliamida incorporando $\mathrm{TiO}_{2}$ e óxido de grafeno (OG), simultaneamente por meio de polimerização interfacial, para a rejeição de sais e fluxo de permeado. Aplicou-se as caracterizações FTIR, XPS e DRX e por meio delas constatou-se a presença do compósito $\mathrm{TiO}_{2}$-OG na superfície da membrana, além de MEV e MFA, que avaliaram a morfologia da superfície como sendo menos rugosa do que a membrana sem aditivos e o ensaio de AC, que foi possível notar a membrana híbrida como sendo mais hidrofílica. O desempenho da membrana em termos de fluxo foi de $62 \mathrm{~L} \cdot \mathrm{m}^{-2} \cdot \mathrm{h}^{-1}$, com $100 \%$ de rejeição de hidrocarbonetos e $97 \%$ de rejeição de sais, indicando melhoria na permeabilidade. Essas melhorias foram obtidas devido ao aumento da carga superficial negativa, hidrofilicidade e diminuição da rugosidade da superfície das membranas, resultantes da adição dos dois aditivos em conjunto.

\section{Considerações Finais}

Sabendo da evolução dos estudos do $\mathrm{TiO}_{2}$ como aditivo em membranas de nanocompósitos poliméricos, nota-se que este material é utilizado principalmente por suas propriedades que trazem melhorias na porosidade e hidroficilidade das membranas, com consequente aumento da permeabilidade, redução de incrustação e capacidade de rejeição de substâncias indesejadas, como corantes e sais, como por exemplo foram encontrados nos estudos, rendimentos em termos de rejeição acima de $94,2 \%$, para a membrana $\mathrm{TFN}$ de $\mathrm{PA} / \mathrm{TiO}_{2}$, chegando a $100 \%$ de rejeição para membranas de $\mathrm{PA}$ com $\mathrm{TiO}_{2} / \mathrm{OG}$. O $\mathrm{TiO}_{2}$ associado com outros aditivos aumentam a performance da membrana quando comparado com a adição de apenas outros aditivos, e a depender do tipo de inserção na membrana pode potencializar até mesmo a redução das características negativas da membrana que se é produzida, por exemplo a introdução de uma camada intermediária de $\mathrm{TiO}_{2}$ em membranas de poliamida contendo óxido de alumínio, as tornou mais hidrofílicas em comparação a mesma membrana sem essa modificação. Fica claro então que este aditivo é altamente eficiente para ser utilizado em conjunto com os processos de separação por membranas, pois foram abordados estudos com diferentes processos, independente da técnica utilizada para a produção de suas nanopartículas ou para a síntese das membranas poliméricas. 


\section{Agradecimentos}

Os autores agradecem ao Grupo de Pesquisa em Tratamentos Avançados de Águas - GRUTAA/UEPB, ao Conselho Nacional de Desenvolvimento Científico e Tecnológico - CNPq e à Coordenação de Aperfeiçoamento de Pessoal de Nível Superior - CAPES pelas bolsas de pesquisa concedidas.

\section{Referências}

Akakuru, O. U., Iqbal, Z. M., \& Wu, A. (2020). $\mathrm{TiO}_{2}$ Nanoparticles: Properties and Applications. In: Wo, A., Ren, W. TiO 2 Nanoparticles: Applications in Nanobiotechnology and Nanomedicine. John Wiley \& Sons, 1-66.

Al-Gamal, A. Q., Falath, W. S., \& Saleh, T. A. (2021). Enhanced efficiency of polyamide membranes by incorporating TiO ${ }_{2}$-Graphene oxide for water purification. Journal of Molecular Liquids, 323, e-114922. https://doi.org/10.1016/j.molliq.2020.114922

Anadão, P. (2010). Ciência e Tecnologia de Membranas. Artliber Editora Ltda., 200 p.

Anan, N. S. M., Jaafar, J., Sato, S., \& Mohamud R. (2015). Titanium dioxide incorporated polyamide thin film composite photocatalytic membrane for Bisphenol A removal. IOP Conf. Ser.: Mater. Sci. Eng. e-1142. . https://doi.org/10.1088/1757-899X/1142/1/012015

Aquino, A. (2011). As Diferenças entre Nanofiltração, Ultrafiltração, Microfiltração e Osmose Reversa. Revista e Portal Meio Filtrante, 10 (53), e-649.

Arribas, P., Khayet, M., García-Payo, M. C., \& Gil, L. (2015). Novel and emerging membranes for water treatment by hydrostatic pressure and vapor pressure gradient membrane processes. In: Basile, A., Cassano, A., Rastogi, N. K. (Ed.). Advances in Membrane Technologies for Water Treatment: Materials, Processes and Applications. 1. ed. Sawston: Woodhead Publishing Series in Energy, 239-286. https://doi.org/10.1016/B978-1-78242-121-4.00008-3

Babu, R. P., O’Connor, K., \& Seeram, R. (2013). Current progress on bio-based polymers and their future trends. Progress in Biomaterials, 2, e-8. https://doi.org/10.1186/2194-0517-2-8

Bai, Y., Mora-Seró, I., De Angelis, F., Bisquert, J., \& Wang, P. (2014). Titanium dioxide nanomaterials for photovoltaic applications. Chemical Reviews, 114, 10095-10130. https://doi.org/10.1021/cr400606n

Baig, M. I., Ingole, P. G., Jeon, J., Hong, S. U., Choi, W. K., \& Lee, H. K. (2019). Water vapor transport properties of interfacially polymerized thin film nanocomposite membranes modified with graphene oxide and GO-TiO2 nanofillers. Chemical Engineering Journal, 373, 1190-1202. https://doi.org/10.1016/j.cej.2019.05.122

Baker, R. W. (2004). Membrane Technology and Applications. (2a ed.), John Wiley \& Sons Inc., 545 p.

Carraher Jr., C. E. (2012). Introduction to Polymer Chemistry. (2a ed.), CRC Press, 545 p.

Colombi, B. L. (2016). Polimerização da Poliamida 6,6: Uma Breve Revisão. Revista Eletrônica de Materiais e Processos, 11 (3), $121-129$.

Cordier, C., Stavrakakis, C., Morga, B., Degrémont, L., Voulgaris, A., Bacchi, A., Sauvade, P., Coelho F., \& Moulin, P. (2020). Removal of pathogens by ultrafiltration from sea water. Environment International, 142, e-105809. https://doi.org/10.1016/j.envint.2020.105809

De Gisi, S., Lofrano, G., Grassi, M., \& Notarnicola, M. (2016). Characteristics and adsorption capacities of low-cost sorbents for wastewater treatment: a review. Sustainable Materials and Technologies, 9, 10-40. https://doi.org/10.1016/j.susmat.2016.06.002

Deopura, B. L. (2008). Polyamide fibers. In: Deopura, B.L., Alagirusamy, R., Joshi, M., \& Gupta, B. (Ed.). Polyesters and Polyamides. Sawston: Woodhead Publishing, 41-61. https://doi.org/10.1533/9781845694609.1.41

Deowan, S. A., Bouhadjar, S. I., \& Hoinkis, J. (2015). Membrane bioreactors for water treatment. In: Basile, A.; Cassano, A.; Rastogi, N. K. (Ed.). Advances in Membrane Technologies for Water Treatment: Materials, Processes and Applications (Woodhead Publishing Series in Energy), Sawston: Woodhead Publishing, 155-184. https://doi.org/10.1016/B978-1-78242-121-4.00005-8

Dinari, M., \& Haghighi, A. (2017). Surface modification of TiO2 nanoparticle by three dimensional silane, coupling agent and preparation of polyamide/modified-TiO2 nanocomposites for removal of $\mathrm{Cr}$ (VI) from aqueous solutions. Progress in Organic Coatings, 110, $24-34$. https://doi.org/10.1016/j.porgcoat.2017.04.044

Galvão, D. F., \& Gomes, E. R. S. (2015). Os processos de separação por membranas e sua utilização no tratamento de efluentes industriais da indústria de laticínios: revisão bibliográfica. Revista do Instituto Laticínios Cândido Tostes, 70 (6), 349-360. https://doi.org/10.14295/2238-6416.v70i6.487

Grimes, C. A., \& Mor. G. K. (2009). TiO 2 Nanotube Arrays: Synthesis, Properties, and Applications. Springer, 358 p.

Isawi, H. (2018). Development of thin-film composite membranes via radical grafting with methacrylic acid/ $\mathrm{ZnO}_{\mathrm{nO}}$ oped TiO $\mathrm{O}_{2}$ nanocomposites. Reactive and Functional Polymers, 131, 400-413. https://doi.org/10.1016/j.reactfunctpolym.2018.08.018

Judd, S. (2010). The MBR book: Principles and applications of membrane bioreactors for water and wastewater treatment. Elsevier, $536 \mathrm{p}$.

Kasvi. (2017). Sistemas Filtração: Princípios e aplicações. https://kasvi.com.br/sistemas-filtracao/

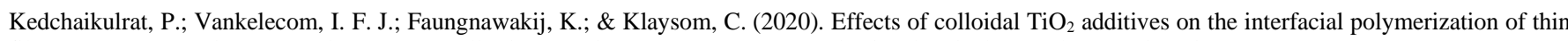
film nanocomposite membranes. Colloids and Surfaces A: Physicochemical and Engineering Aspects, 601, e-125046. https://doi.org/10.1016/j.colsurfa.2020.125046 
Kim, S. H., Kwak, S., Sohn, B., \& Park, T. H. (2003). Design of TiO2 nanoparticle self-assembled aromatic polyamide thin-film-composite (TFC) membrane as an approach to solve biofouling problem. Journal of Membrane Science, 211, 157-165. https://doi.org/10.1016/S0376-7388(02)00418-0

Lee, H. S., Im S. J., Kim, J. H., Kim, H. J., Kim, J. P., \& Min B. R. (2008). Polyamide thin-film nanofiltration membranes containing TiO 2 nanoparticles. Desalination, 219, 48-56. https://doi.org/10.1016/j.desal.2007.06.003

Li Y., Cao Y., Wang M., Xu Z., Zhang H., Liu X., \& Li Z. (2018). Novel high-flux polyamide/TiO 2 composite nanofiltration membranes on ceramic hollow fibre substrates. Journal of Membrane Science, 565, 322-330. https://doi.org/10.1016/j.memsci.2018.08.014

Li, Y., Zhang, S., Yu, Q., \& Yin, W. (2007). The effects of activated carbon supports on the structure and properties of TiO ${ }_{2}$ nanoparticles prepared by a solgel method. Applied Surface Science, 253, 9254-9258. https://doi.org/10.1016/j.apsusc.2007.05.057

Liderfelt, J., \& Royce, J. (2017). Filtration Principles. In: Jagschies, G.; Lindskog, E.; Łącki, K.; \& Galliher, P. (Ed.). Biopharmaceutical Processing: Development, Design, and Implementation of Manufacturing Processes. Amsterdam: Elsevier, 2017. 279 - 293. https://doi.org/10.1016/B978-0-08-1006238.00014-1

Lilane, A., Saifaoui, D., Hariss, S., Jenkal, H., \& Chouiekh, M. (2020). Modeling and simulation of the perform reverse osmosis membrane. Materials Today: Proceedings, 24 (1), 114-118. https://doi.org/10.1016/j.matpr.2019.07.694

Liu, W., Cao Y., Li, Y., Xu Z., Li, Z., Wang, M., \& MA X. (2019). High-performance polyamide/ceramic hollow fiber TFC membranes with TiO 2 interlayer for pervaporation dehydration of isopropanol solution. Journal of Membrane Science, 576, 26-35. https://doi.org/10.1016/j.memsci.2019.01.023

Matsuura, T. (1993). Synthetic Membranes and Membrane Separation Processes. CRC Press, 480 p.

McKeen, L. W. (2019). Polyamides (Nylons). In: McKeen, L. W. The Effect of UV Light and Weather on Plastics and Elastomers. 4. ed. Norwich: William Andrew Applied Science Publishers, 187-227. https://doi.org/10.1016/B978-0-12-816457-0.00007-1

Mulder, M. (1996). Basic Principles of Membrane Technology. (2a ed.), Kluwer Academic Publishers, 564 p.

Mutuma, B. K., Shao, G. N., Kim, W. D., \& Kim, H. T. (2015). Sol-gel synthesis of mesoporous anatase-brookite and anatase-brookite-rutile TiO 2 nanoparticles and their photocatalytic properties. Journal of Colloid and Interface Science, 442, 1-7. https://doi.org/10.1016/j.jcis.2014.11.060

Ngo, T. H. A., Nguyen, D. T., Do, K. D., Nguyen, T. T. M., Mori, S., \& Tran, D. T. (2016). Surface modification of polyamide thin film composite membrane by coating of titanium dioxide nanoparticles. Journal of Science: Advanced Materials and Devices, 1 (4), 468-475. https://doi.org/10.1016/j.jsamd.2016.10.002

Paz, R. A., Leite, A. M. D., Araújo, E. M., Melo, T. J. A., Barbosa, R., \& Ito, E. N. (2008). Nanocompósitos de poliamida 6/argila organofílica: efeito do peso molecular da matriz na estrutura e propriedades mecânicas e termomecânicas. Polímeros, 18 (4), 341-347.

Pham, T., \& Dang, T. N. (2019). Microcystins in Freshwater Ecosystems: Occurrence, Distribution, and Current Treatment Approaches. In: BUI, X., Chiemchaisri, C., Fujioka, T., Varjani, S. (Ed.). Water and Wastewater Treatment Technologies, Energy, Environment, and Sustainability. Springer, 15-36. https://doi.org/10.1007/978-981-13-3259-3_2

Rajaeian, B., Rahimpour, A., Tade, M. O., \& Liu, S. (2013). Fabrication and characterization of polyamide thin film nanocomposite (TFN) nanofiltration membrane impregnated with $\mathrm{TiO}_{2}$ nanoparticles. Desalination, 313, 176-188. https://doi.org/10.1016/j.desal.2012.12.012

Ribeiro, P. C., Costa, A. C. F. M., Kiminami, R. H. G. A., Sasaki, J. M., \& Lira, H. L. (2010). Caracterização estrutural e morfológica de nanocristais de TiO pelo método pechini. Revista Eletrônica de Materiais e Processos, 5 (3), 58-64.

Santos, L. M. (2017). Síntese e caracterização de TiO2 com modificações superficiais para aplicação em fotocatálise heterogênea. Tese de Doutorado em Química da Universidade Federal de Uberlândia, 135 p.

Shao, F., Xu, C., Ji, W., Dong, H., Sun, Q., Yu, L., \& Dong, L. (2017). Layer-by-layer self-assembly TiO2 and graphene oxide on polyamide reverse osmosis membranes with improved membrane durability. Desalination, 423, 21-29. https://doi.org/10.1016/j.desal.2017.09.007

Shen, H., Wang, S., Xu, H., Zhou, Y., \& Gao, C. (2018). Preparation of polyamide thin film nanocomposite membranes containing silica nanoparticles via an in-situ polymerization of SiCl4 in organic solution. Journal of Membrane Science, 565, 145-156. https://doi.org/10.1016/j.memsci.2018.08.016

Singh R., Satyannarayana K.V.V., Vinoth Kumar R., \& Ganesh Moorthy I. (2020) Membrane Technology in Bioprocess Engineering. In: Jerold M., Arockiasamy S., Sivasubramanian V. (Ed.). Bioprocess Engineering for Bioremediation. The Handbook of Environmental Chemistry. Cham: Springer, 1-26. https://doi.org/10.1007/698_2020_505.

Susuki, F. F. M., Paraíso, P. R., Almeida, P. H. S., Ito A., \& Bergamasco, R. (2016). Produção e caracterização de membranas poliméricas, usando os aditivos cloreto de potássio e de lítio, em condições idênticas. Engevista, 8 (1), 142-157. https://doi.org/10.22409/engevista.v18i1.711

Tayefeh, A, Mousavi, S. A., Wiesner, M., \& Poursalehi, R. (2015). Synthesis and Surface Characterization of Magnetite-Titania Nanoparticles/Polyamide Nanocomposite Smart RO Membrane. Procedia MaterialsScience, 11, 342-346. https://doi.org/10.1016/j.mspro.2015.11.114

Teegarden, D. M. (2004). Polymer Chemistry: Introduction to an Indispensable Science. Arlington: National science teachers association, 280 p.

Urper-Bayram, G. M., Bossa, N., Warsinger, D. M., Koyuncu, I., \& Wiesner, M. (2020). Comparative impact of $\mathrm{SiO}_{2}$ and TiO ${ }_{2}$ nanofillers on the performance of thin-film nanocomposite membranes. Journal of Applied Polymer Science, 137 (44), e-49382. https://doi.org/10.1002/app.49382

Wypych, G. (2016). PA-6 polyamide-6. In: Wypych, G. Handbook of Polymers. (2a ed.), ChemTec Publishing, $215-220$.

Yong, M., Zhang, Y., Sun, S., \& Liu, W. (2019). Properties of polyvinyl chloride (PVC) ultrafiltration membrane improved by lignin: hydrophilicity and antifouling. Journal of Membrane Science, 575, 50-59. https://doi.org/10.1016/j.memsci.2019.01.005 
Research, Society and Development, v. 10, n. 8, e48110817434, 2021

(CC BY 4.0) | ISSN 2525-3409 | DOI: http://dx.doi.org/10.33448/rsd-v10i8.17434

Zarshenas, K., Jiang, G., Zhang J., Jauhar M. A., \& Chen Z. (2020). Atomic scale manipulation of sublayer with functional $\mathrm{TiO}_{2}$ nanofilm toward highperformance reverse osmosis membrane. Desalination, 480, e-114342. https://doi.org/10.1016/j.desal.2020.114342

Zhao, B., Chen, F., Huanga, Q., \& Zhanga, J. (2009). Brookite $\mathrm{TiO}_{2}$ nanoflowers. Chemical Communications, 5115 (34), $5115-5117$. https://doi.org/10.1039/B909883F 